\title{
Application of dynamic resistance measurement in the contact ablation assessment of high voltage $\mathrm{SF}_{6}$ circuit breaker
}

\author{
Jing-Gang Yang \\ State Grid Jiangsu Electric Power Company Research Institute, \\ Nanjing 211103, China \\ Ya-Kui Liu ${ }^{\dagger}$ \\ State Key Lab of Electrical Insulation and Power Equipment \\ $X i$ 'an Jiaotong University \\ $X i^{\prime}$ an 710049, China \\ †E-mail:lykppqq@163.com \\ Shan Gao, Ke Zhao and Hong-Tao Li \\ State Grid Jiangsu Electric Power Company Research Institute, \\ Nanjing 211103, China \\ Guo-Gang Zhang \\ State Key Lab of Electrical Insulation and Power Equipment \\ $X i$ 'an Jiaotong University \\ Xi'an 710049, China \\ E-mail: ggzhang@mail.xjtu.edu.cn
}

\begin{abstract}
High voltage circuit breakers are most important protective device in power system. The contact ablation may cause the failure of circuit breaker and endangers the safety of the power system. Dynamic resistance measurement (DRM) is considered to be an effective assessment technique for contact ablation when the circuit breaker has a lower breaking speed. However, the resistance curve at higher rated speed during opening operation of high voltage circuit breaker is hardly to identify the main contact part as well as the arcing contact part. In this paper, a DRM characteristic analyzer has been used to collect the curves of resistance vs. time from an experimental prototype of high voltage SF6 circuit breaker during opening and closing operations. Based on the analysis of the experimental results, both the travel extracted from the curve during closing operation and the average resistance extracted from the curve during opening operation can be used to implement the condition assessment of the contact ablation, and the combined data show more details about the main contacts and arcing contacts of high voltage circuit breaker.
\end{abstract}

Keywords: High voltage circuit breaker; dynamic resistance measurement; contact ablation; condition assessment. 


\section{Introduction}

A high voltage circuit breaker is the protective device of electrical system that automatically interrupts the current flowing through it when that current exceeds a certain value for a specified period of time. Contact system is the most important part of circuit breaker. The condition of contact system has a direct impact on the interrupting performance and insulation of the extinguishing chamber. When the circuit breaker breaks the load or the fault current, the arcing contact bears all the current and generates electric arc between the stationary arcing contact and the movable arc contact. The electric arc will cause some loss of arcing contact. If the loss is so serious that the breaker cannot tolerate, which makes the arc contact unable to be separated before main contact, it will cause the failure of circuit breaker and endangers the safety of the power system.

The dynamic resistance measurement (DRM) was developed to assess the condition of the arcing contacts without dismantling the breaker. And the contact resistance between the moving and static contact can be measured [1,2] by a high injection current [3], it gets the dynamic resistance-time curve and travel-time curve, with the measurement on the breaker contact speed [4,5], and it can calculate the arc contact resistance as well as the travel from the arc contact to the main contact, which is used to diagnostic the ablation condition $[6,7]$.

All the previous analyses only focus on the DRM during the opening operation at low breaking speed, which researchers hold the view that the DRM during closing operation is not generally useful since the measurement must be performed during a transient state. However, the curve during closing operation is more effective than the curve during opening operation in the condition of the circuit breaker is hardly to low its breaking speed.

In order to check the efficiency and accuracy of the method, an experimental prototype of LW30-252 $\mathrm{SF}_{6}$ circuit breaker is used. Figure 1 shows the circuit model, in which DRM analyzer is used to collect data and super capacitor is used to provide direct current.

\section{DRM During the Opening Operation}

Major headings should be typeset in boldface with the first letter of important words capitalized.

The circuit breaker with a spring operating mechanism is used in this test, which is not easy to adjust the breaking speed. The average closing speed is $3 \mathrm{~m} / \mathrm{s}$, while the average opening speed is $9 \mathrm{~m} / \mathrm{s}$. Only a small amount of mechanical loss can be achieved and the arc loss in the experiment can be 
neglected because the breaker is off-line. The travel which arc contact beyond main contact is $20 \mathrm{~mm}$. Figure 2 shows the arc extinguishing chamber.

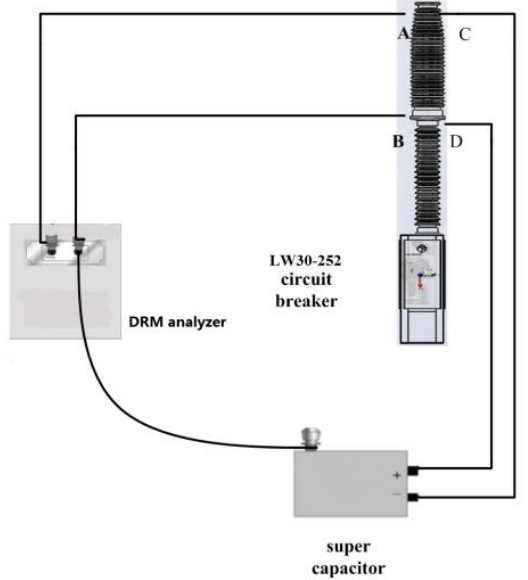

Fig.1. DRM test circuit model;

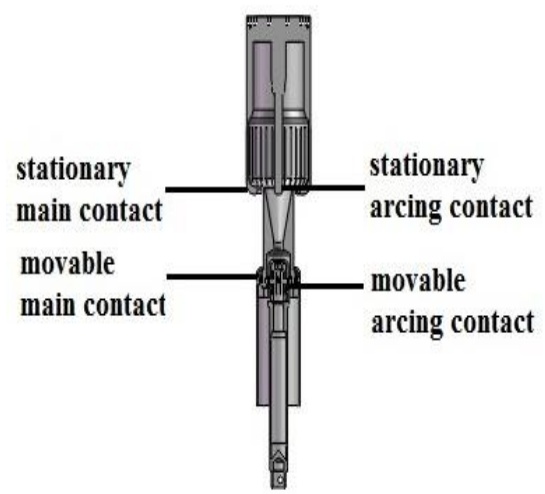

Fig.2. Arc extinguishing chamber

In opening operation, the movable main contact is firstly separated from the stationary main contact, and the movable arc contact is separated from the stationary arc contact after a period of time. Therefore, the dynamic resistance curve is divided into main contact part and arc contact part. In the main contact part, both the main contact and the arc contact make touch, so the resistance curve is influenced by the contact resistance of the main contact and the arc contact, and they are considered to be parallel. When the process enters the arc contact part, it is considered that the arc contact resistance alone is affecting the resistance curve of the arc contact stage, as a result that the main contact has been separated.

Figure 3 shows the curve during opening operation that has been collected by the characteristic analyzer in the experimental prototype, in which the horizontal axis represents the distance, and the vertical axis represents the resistance.

It can be observed that the curve has several peaks and valleys, the last peak before the general trend of the curve tends to be stable is considered the ending of main contact part and the beginning of the arcing contact part according to the rule. But in this case, the travel which arc contact beyond main contact is only $10 \mathrm{~mm}$, which is far below the normal condition. It may lead people wrongly believe that the contact has been seriously ablated. 


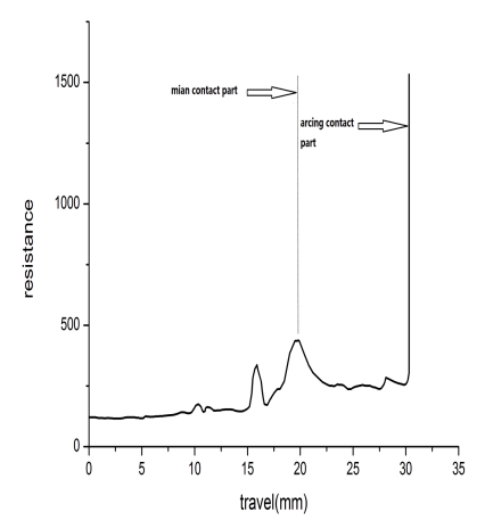

Fig.3. DRM curve during opening operation;

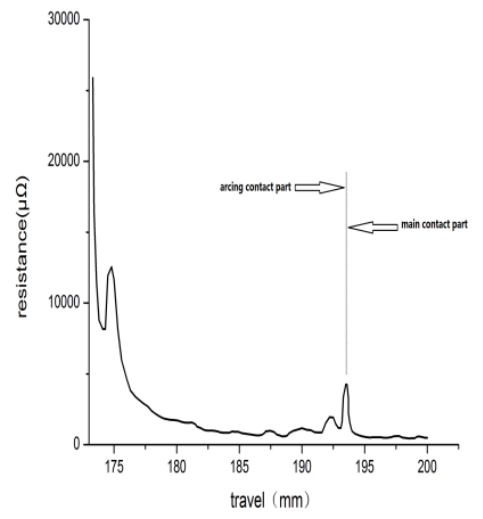

Fig.4. DRM curve during closing operation;

Table 1 Parameter comparison between closing and opening operation

\begin{tabular}{lccccc}
\hline & $l_{\mathrm{m}}[\mathrm{mm}]$ & $l_{\mathrm{r}}[\mathrm{mm}]$ & $l_{\mathrm{b}}[\mathrm{mm}]$ & $R_{\mathrm{m}}[\mu \Omega]$ & $R_{\mathrm{a}}[\mu \Omega]$ \\
\hline Opening operation & 19.3 & 30.3 & 11 & 126.49 & 258.54 \\
Closing operation & 6.5 & 27 & 20.5 & 449.62 & 875.36 \\
\hline
\end{tabular}

\section{DRM During the Closing Operation}

Figure 4 shows the curve during closing operation that has been collected by the characteristic analyzer in the experimental prototype, in which the horizontal axis represents the distance, and the vertical axis represents the resistance.

Contrary to opening operation, movable arc contact first touches stationary arc contact during closing operation, and then the main contact occurs, which resulted in the process steps into arc contact part firstly, and then steps into the main contact part.

There is no contact between the moving contact and static contact before the closing operation, and the resistance is infinite at the time. Along with the closing operation, the contact begins contact, and the resistance is no longer infinite any more. This point is taken as the beginning of the arc contact part. After a series of oscillations, the curve reaches the final peak, after which the curve becomes small and tends to be stable. This is taken as the ending of arc contact part and the beginning of main contact part, the corresponding travel be considered to be the travel that arc contact beyond main contact. After that, the travel is considered to be main contact travel.

In this curve, the main contact part and the arcing contact part can be easily identified, the travel which arc contact beyond main contact is $20.5 \mathrm{~mm}$. 


\section{Discussions}

In this paper, the travel and resistance are extracted as the characteristic parameters, it's because that:

(1) The travel from the arc contact to the main contact is an important parameter to evaluate the ablation condition of the contact. If the travel is too short, the current may be prevented from transferring the current from the main contact to the arc contact, which may results in the arc burn on the main contact and cause the arc extinguishing chamber to lose its effect;

(2) The average resistance can reflect the condition of the contact. Once the resistance value is too large, which means the contact has been ablated, the contact between the moving and static arc contact is poor.

The travel include main contact travel $\left(l_{\mathrm{m}}\right)$, arc contact travel $\left(l_{\mathrm{r}}\right)$ and travel which arc contact beyond the main contact $\left(l_{\mathrm{b}}\right)$. The resistance includes the average resistance of the main contact part $\left(R_{\mathrm{m}}\right)$ and the average resistance of the arc contact part $\left(R_{\mathrm{a}}\right)$, as shown in table 1 .

From the table we can easily find that,

(1) The travel is different. The travel which arc contact beyond the main contact during closing operation is larger than the travel during opening operation. According to the structure of circuit breaker, the larger one is considered effective while the other one is considered to be false. This phenomenon is considered to be caused by partial touch of contact due to high touching speed during opening operation.

(2) The resistance is different. The resistance during closing operation is larger than the resistance during the opening operation. It is attributed to the speed of the contact movement and the current which flow through the contact are different.

\section{Conclusions}

In summary, the travel extracted from the curve during closing operation and the average resistance extracted from the curve during opening operation are considered to be the characteristic parameters to diagnostic the contact ablation degree of high voltage circuit break under. Compared with opening speed, closing speed is lower so that it is more beneficial to analyze.

\section{Acknowledgments}

This work was supported by Project Program of State Grid Corporation of China and the National Natural Science Foundation of China (51407026). 


\section{References}

1. Z. Stanisic. International Conference and Exhibition on Innovative Smart Grid Technologies. Manchester, Europe: IEEE, (2011), p. 5-7

2. Z. Stanisic, and R. Neimanis. Conference Record of the 2010 IEEE International Symposium on Electrical Insulation (ISEI). [S.1.]: IEEE, (2010), p. 1, 4, 6-9

3. T. Cheng, W. Zhu, and G. Jin. International Conference on Electricity Distribu-tion. Shenzhen, China: CICED,( 2014), p.1477-1481

4. M. Landry, A. Mercier and G. Ouellet. Transmission and Distribution Conference and Exhibition. [S.1.]:IEEE,( 2006), p. 1002-1009

5. M. Landry, O. Turcotte, F. Brikci. IEEE Transactions on Power Delivery, 23 (2008), p. 710-716

6. G. Xu, J. Zhang and J. Qian. High voltage circuit breaker: theory and application (Tsinghua University Press, China, 2000)

7. Y. Chen, W. Xu and W. YUAN. High Voltage Engineering, 39 (2013), p. 937-942 